\title{
Analogs of farnsyl diphosphate alter CaaX substrate specificity and reactions rates of protein farnesyltransferase
}

Benjamin C. Jennings ${ }^{\mathrm{a}}$, Amy M. Danowitz ${ }^{\mathrm{b}}$, Yen-Chih Wang ${ }^{\mathrm{c}}$, Richard A. Gibbs ${ }^{\mathrm{d}, \dagger}$, Mark D. Distefano $^{\mathrm{c}, \mathrm{e}}$, Carol A. Fierke ${ }^{\mathrm{a}, \mathrm{f}_{*}}$

${ }^{a}$ Department of Chemistry, University of Michigan, Ann Arbor, MI, 48109, USA

${ }^{\mathrm{b}}$ Department of Chemistry and Biochemistry, Mercyhurst University, Erie, PA, 16546, USA

'Department of Department of Chemistry, University of Minnesota, Minneapolis, MN 55455, USA

${ }^{\mathrm{d}}$ Department of Medicinal Chemistry and Molecular Pharmacology, Purdue University, West

Lafayette, IN 47907, USA

${ }^{e}$ Department of Medicinal Chemistry, University of Minnesota, Minneapolis, MN 55455, USA

${ }^{\mathrm{f}}$ Department of Biological Chemistry, University of Michigan, Ann Arbor, MI, 48109, USA

†Deceased

*Corresponding author: fierke@umich.edu, 930 North University Avenue, Ann Arbor, MI 481091055. Phone, (734) 936-2678; fax, (734) 647-4865.

Keywords:

1. Protein farnesylation

3. Click chemistry

5. Post-translational modification

\section{Protein prenylation}

4. Substrate analogs

\section{Abstract:}

Attempts to identify the prenyl-proteome of cells or changes in prenylation following drug treatment have used "clickable" alkyne-modified analogs of the lipid substrates farnesyl- and geranylgeranyl-diphosphate (FPP and GGPP). We characterized the reactivity of four alkynecontaining analogs of FPP with purified protein farnesyltransferase and a small library of dansylated peptides using an in vitro continuous spectrofluorimetric assay. These analogs alter prenylation specificity and reactivity suggesting that in vivo results obtained using these FPP analogs should be interpreted cautiously.

Graphical abstract:

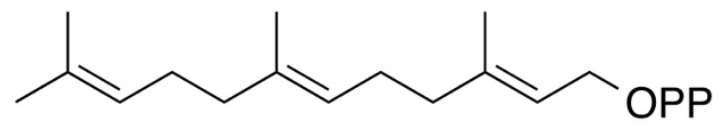

Farnesyl diphosphate (FPP) endogenous substrate

VS.

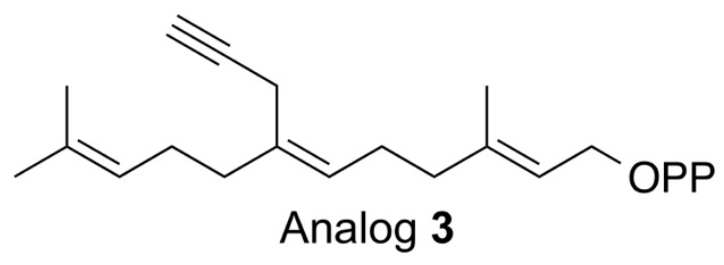

Farnesyltransferase + dansyl-GCVa $2 \mathrm{~S}$

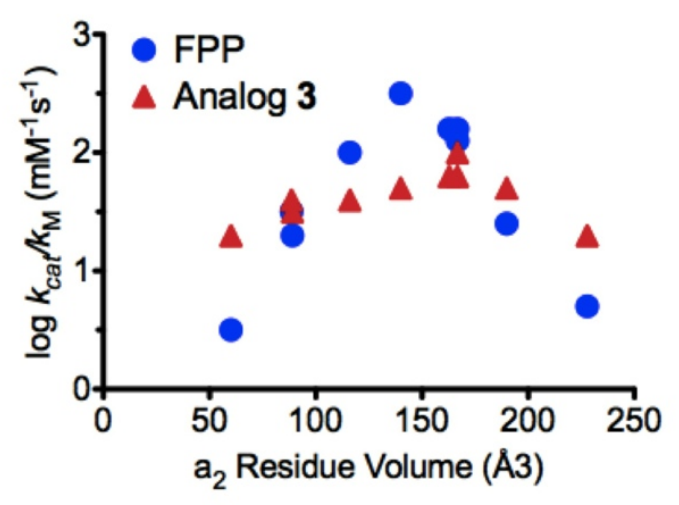


Text:

Protein farnesyltransferase (FTase) is a heterodimeric zinc metalloenzyme that catalyzes the covalent attachment of a 15-carbon isoprenoid unit from farnesyl diphosphate (FPP) to a cysteine four residues from the C-terminus of a protein substrate. ${ }^{1}$ Farnesylation is required for the proper localization of many proteins involved in signal transduction, including Ras. ${ }^{2}$ Mutated Ras has been found in over $16 \%$ of all human cancers ${ }^{3}$, and the initial step of Ras farnesylation is sufficient for conferring transforming potential. ${ }^{4}$ Therefore, FTase inhibitors (FTIs) were developed, which displayed anti-cancer properties and have been tested in clinical trials. ${ }^{5}$ However, in FTI-treated cells, Ras can be alternatively prenylated by a related enzyme, GGTase-I (geranylgeranyltransferase-I). ${ }^{6}$ This finding suggests that FTIs may inhibit the farnesylation of other proteins involved in oncogenesis and proliferation of cancer cells. Identifying these proteins would aid the development of novel strategies for treating cancer.

Previous studies have shown that protein substrates of FTase share a common $\mathrm{Ca}_{1} \mathrm{a}_{2} \mathrm{X}$ motif at their C-termini where "C" is a cysteine residue, " $\mathrm{a}_{1}$ " and " $\mathrm{a}_{2}$ " are frequently aliphatic amino acids and "X", in the case of an FTase substrate, is generally a serine, methionine, glutamine or alanine. ${ }^{7,8}$ While this paradigm has been useful for predicting some of the endogenous targets of farnesylation, ${ }^{9}$ it does not fully encompass the range of potential protein substrates. For example, structure-based modeling predicted a novel class of substrates with negatively charged $\mathrm{C}$-terminal residues (CxxD and $\mathrm{CxxE}$ ) that was experimentally verified. ${ }^{10}$ Additionally, several studies have indicated that amino acid residues upstream of the cysteine residue may also contribute to substrate selectivity. ${ }^{11}$ Due to the limitations of the CaaX paradigm and the large number of proteins in a typical mammalian cell that could potentially be farnesylated, all of the endogenous targets of FTase have yet to be identified. A strategy for the global discovery of proteins modified by FTase would be beneficial for determining the targets of FTIs and for characterizing the full complement of farnesylated proteins.

One strategy for the in cellulo identification of post-translationally modified proteins takes advantage of bio-orthogonal "click" chemistry between an azide and an alkyne and has been applied to the study of farnesylation. ${ }^{12}$ As FPP analogs can be easily synthesized, several groups have developed alkyne- or azide- modified FPP derivatives. ${ }^{13-18}$ FTase uses these derivatives as a substrate in place of endogenous FPP in cellulo, resulting in the covalent modification of native protein substrates. Tagged proteins are then captured and identified by mass spectrometry. Similar methods based on either a biotin-modified isoprenoid and mutant transferases ${ }^{19}$ or antigenic isoprenoid derivatives that can be detected via western blotting techniques ${ }^{20}$ have also been employed for this purpose. Several of these FPP derivatives have been used to identify FTase protein substrates from cells. ${ }^{13,16,17,21,22}$ Some of the 
results from these initial experiments have been surprising. For example, several studies using different analogs have identified annexin proteins (a class of proteins that do not contain a CaaX motif, rather a-CGGDD C-terminus) as potential FTase substrates. ${ }^{13}$ Additionally, several of the analogs tested have not detected known farnesylated proteins such as transducin $\gamma$ (CVIS). ${ }^{13,16}$ Finally, different analogs have identified partially overlapping but distinct sets of prenylated proteins. Some variability is expected from the complex processing during click-conjugation, purification, and mass spectrometry; from differences in protein abundance, concentration, and location with different cell types; and from differences in the reactivity of prenyltransferases with each analog. However, it is also possible that the location of the alkyne modification in the analog may influence which proteins each analog can modify.

This information led to the hypothesis that the structures of the various analogs affect the substrate selectivity of FTase. Consistent with this, the crystal structure of FPP-bound FTase shows that the second isoprenoid unit of FPP interacts with the amino acid residue at the $\mathrm{a}_{2}$ position of the bound peptide substrate. ${ }^{23}$ Similarly, biochemical studies demonstrate that the steady-state rate constants of farnesylation depend on both the size of the residue at the $\mathrm{a}_{2}$ position and modifications on the isoprenoid units of FPP analogs. ${ }^{24-26}$ Based on these data, we hypothesized that different FPP analogs would alter the peptide selectivity of FTase based on the size of the residue in the $\mathrm{a}_{2}$ position. To test this hypothesis, four alkyne-modified FPP analogs, analog $\mathbf{1}^{27}, \mathbf{2}$ and $\mathbf{3}^{28}$, and $\mathbf{4}^{14}$ (Figure 1), were synthesized, as previously reported. The affect of these analogs on FTase peptide sequence specificity was then determined by comparing their in vitro kinetic activity to that of FPP for catalyzing farnesylation of a library of peptides with varying CaaX sequences. ${ }^{29}$

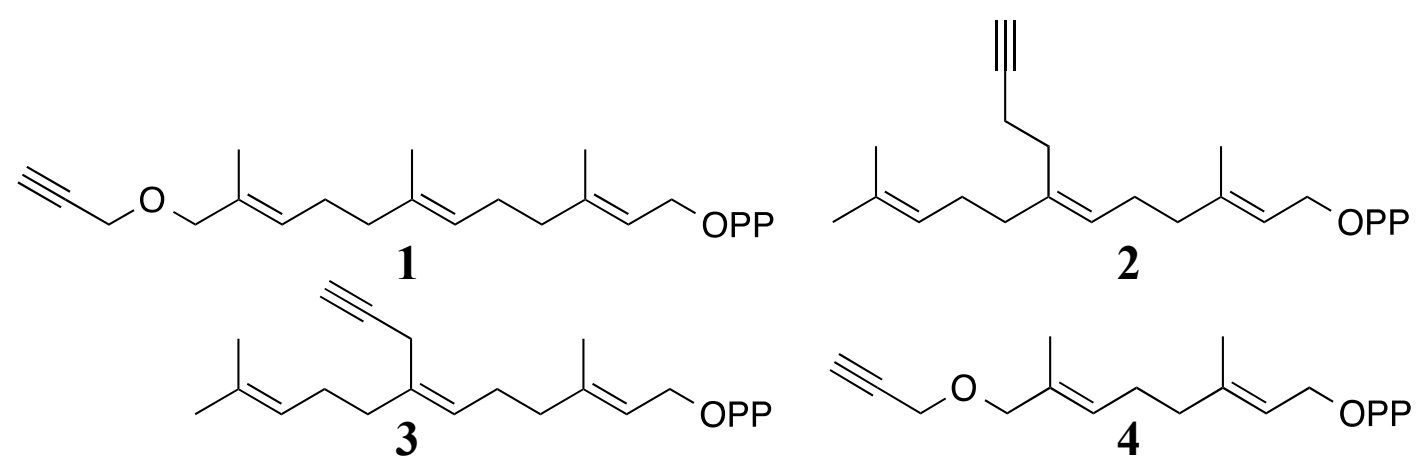

Figure 1. Synthetic alkyne-modified FPP analogs.

Initially, we assayed a peptide library of the form dansyl-GCVa $\mathrm{Cl}_{2} \mathrm{~S}$ where non-ionizable side chains were varied at the $a_{2}$ position. Previous work using FPP demonstrated that when neutral residues were varied in the $\mathrm{a}_{2}$ position, there was a correlation between the value of $k_{c a t} / K_{\mathrm{M}}$ and the volume of the amino acid side chain; the most efficient reactivity was observed with medium-sized 
residues at the $\mathrm{a}_{2}$ position (residue volume $\left.=140 \AA^{3}\right){ }^{24}$ Here, we compared the value of $k_{\text {cat }} / K_{\mathrm{M}}$ with FPP to those obtained using analogs $\mathbf{1}$ - $\mathbf{4}$ to determine if the modified structures alter substrate selectivity. These data demonstrate that the structure of the FPP analog alters the dependence of the FTase activity on the size of the residue at the $\mathrm{a}_{2}$ position (Fig. 2).
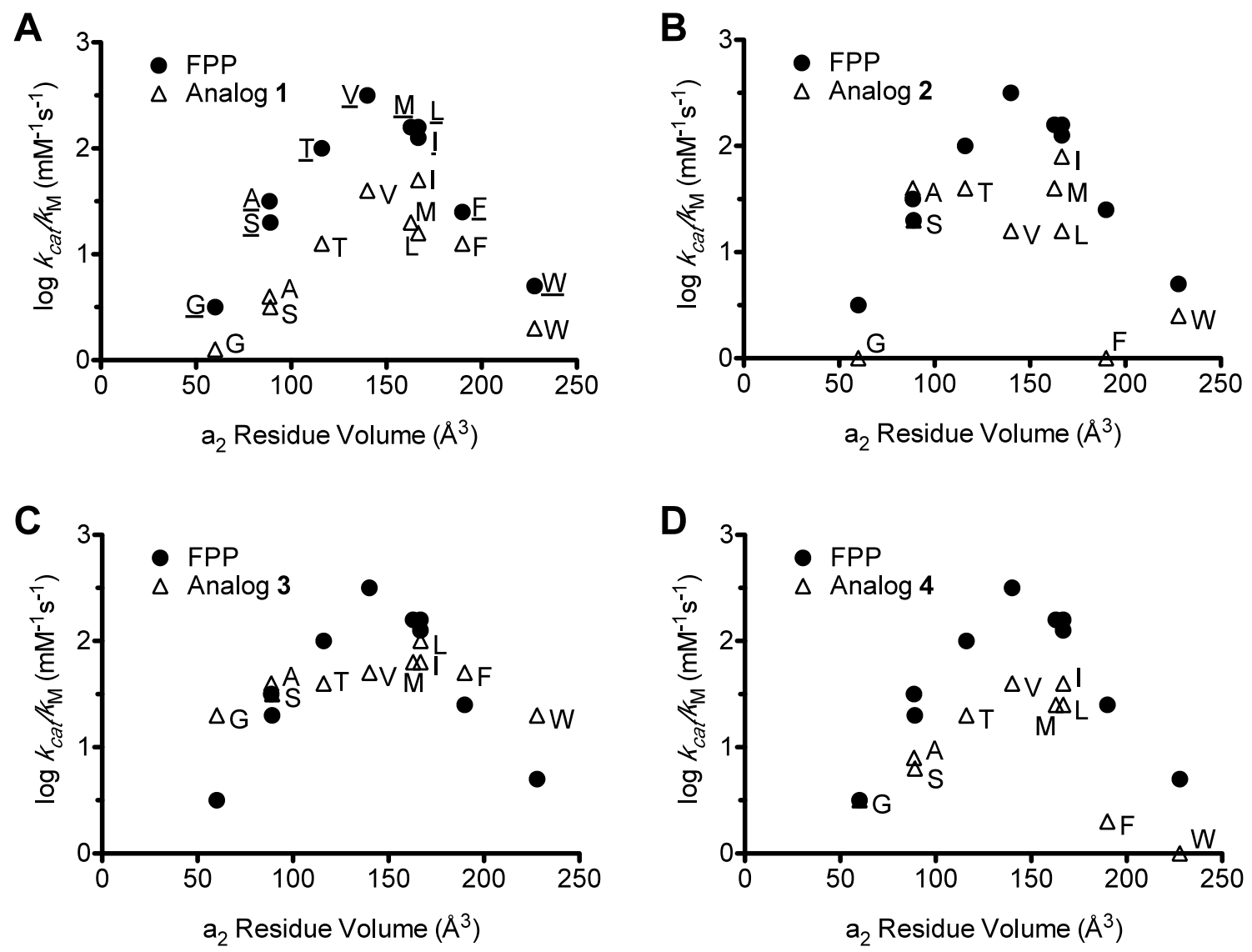

Figure 2. Reactivity of FPP or alkyne-modified FPP analogs with FTase and dansyl-GCVa $\mathrm{S}_{2} \mathrm{~S}$ peptides. The steady-state rate parameters $\left(k_{\mathrm{cat}} / K_{\mathrm{M}}\right)$ were determined using a linear fit of the peptide concentration-dependence of the initial rates of farnesylation using a previously established method. ${ }^{30}$ The side chain volumes (in $\AA^{3}$ ) for the amino acids (labeled by one letter code) used in the $\mathrm{a}_{2}$ position were Gly (60.1), Ala (88.6), Ser (89.0), Thr (116.1), Val (140.0), Met (162.9), Ile (166.7), Leu (166.7), Phe (189.9), and Trp (227.8) as reported previously. ${ }^{31}$

Analog 1 does not have an additional bulky group incorporated within the second isoprenoid unit. As predicted, this analog displayed the characteristic "pyramid" reactivity pattern with FTase as observed for FPP, although the maximal activity ( $a_{2}=$ valine) is decreased (Fig. 2.A). When using analogs $\mathbf{2}$ and $\mathbf{3}$, both of which have the alkyne moiety appended to the second isoprenoid unit as a branch, a different selectivity pattern emerged. We found the reactivity of FTase with $\mathbf{3}$, which contains a propargyl group, is more promiscuous than with FPP, with modest discrimination $(\leq 4$-fold) 
based on the size of the $a_{2}$ residue (Fig. 2.C). This indicates that using analog $\mathbf{3}$ in endogenous studies may lead to capturing a wider variety of potential protein substrates of farnesylation. Analog 2, which incorporates a homopropargyl substituent, displayed a unique pattern where the value of $k_{\text {cat }} / K_{\mathrm{M}}$ varied little for peptide substrates with $\mathrm{a}_{2}$ position side chain volumes between 80-170 $\AA^{3}$. Analog 2,

however, was ineffective at modifying substrates with $\mathrm{a}_{2}$ residue side chain volumes either smaller than $80 \AA^{3}$ or larger than $170 \AA^{3}$. Substrates with a 2 residues in these size ranges exhibited more than a 10 fold reduction in rate relative to peptide substrates with midsized $\mathrm{a}_{2}$ side chain volumes (Fig. 2.C).

Analog 4, structurally similar to 1 but one isoprenoid unit shorter, displayed selectivity similar to $\mathbf{1}$ for residue volumes less than $170 \AA^{3}$ (Fig 2.D). However, the reactivity with peptides with bulky a sides chains (Phe, Tyr, and Trp) was decreased.

In light of this evidence that alkyne modifications affect the peptide selectivity of FTase at the $\mathrm{a}_{2}$ position, we examined if the differences in structure of the analogs affected reactivity with sequences from protein substrates that were previously identified from cells using analogs. To this end, a library of the form dansyl-TKCXxx was designed with sequences from proteins that were previously identified with sequences that either fit or do not fit the CaaX paradigm as well as sequences from prenylated proteins that have not been identified using analogs. Since this peptide library represents biological substrates, there is significant variation in sequence (Table 1) relative to Figure 2. Correspondingly, there is significant variation in observed reactivity for differences in the upstream sequence (TKCaaX vs GCaaX) $)^{11}$ and in the identity of the terminal $\mathrm{a}_{2} \mathrm{X}$ residue pairs, as previously observed. ${ }^{24}$

\begin{tabular}{|c|c|c|c|c|c|c|}
\hline Peptide $^{\mathrm{a}}$ & Derived from & FPP & Analog 1 & Analog 2 & Analog 3 & Analog 4 \\
\hline CGGDD & Annexin A2 and A3 & $<0.1$ & $<0.1$ & $<0.1$ & $<0.1$ & $<0.1$ \\
\hline CVLL & Cdc42 & $<0.2 \pm 0.01^{\mathrm{c}}$ & $<0.1$ & $<0.1$ & $<0.1$ & $<0.1$ \\
\hline CSVL & RAB18 & $<0.2 \pm 0.01^{\mathrm{c}}$ & $<0.1$ & $<0.1$ & $<0.1$ & $<0.1$ \\
\hline CCIQ & $\begin{array}{c}\text { Protein tyrosine } \\
\text { phosphatase PTPCAAX1 }\end{array}$ & $6.6 \pm 0.4$ & $4 \pm 1$ & $<0.1$ & $<0.1$ & $32 \pm 3$ \\
\hline CVLS & H Ras & $47 \pm 9$ & $1.6 \pm 0.1$ & $2.0 \pm 0.4$ & $19 \pm 5$ & $24 \pm 1$ \\
\hline CVIS & Transducin $\gamma$ & $59 \pm 7$ & $10.8 \pm 0.6$ & $41 \pm 12$ & $41 \pm 3^{\mathrm{b}}$ & $24 \pm 1$ \\
\hline CTIQ & Rhes & $4.9 \pm 0.9$ & $1.8 \pm 0.01$ & $12.1 \pm 0.8^{\mathrm{b}}$ & $<0.1$ & $28 \pm 3$ \\
\hline
\end{tabular}

Table 1. Steady state rate parameters for the reaction catalyzed by FTase between FPP analogs and peptides substrate. All of the listed values are $k_{\text {cat }} / K_{\mathrm{M}}\left(\mathrm{mM}^{-1} \mathrm{~s}^{-1}\right)$. These assays were performed and steady-state rate parameters $\left(k_{\mathrm{cat}} / K_{\mathrm{M}}\right)$ were determined as previously reported. ${ }^{30}{ }^{\mathrm{a}} \mathrm{All}$ peptides in this series take the form of dansyl-TKxxx or dansyl-TKxxxx for annexin. ${ }^{\mathrm{b}}$ These peptides were found to cause substrate inhibition at higher concentrations. These values represent lower boundaries of the rates for these peptides. ${ }^{\mathrm{c}}$ These peptides have previously been reported as single turnover peptides. ${ }^{30}$ Value \pm S.E.M. 
The first set of substrates tested were those that were identified in cellulo using one or more of the analogs, but that were not predicted as FTase multiple turnover substrates. Annexin proteins were reported as FTase substrate from cells in studies using either analog $4^{16}$ or an FPP-azido analog. ${ }^{13}$ The C-terminus of annexin A2 and A3 is-CGGDD; thus these proteins are not predicted as FTase substrates since the cysteine at the $\mathrm{C}$-terminus is 5 amino acids from the end. We demonstrated a lack of modification of annexin peptides catalyzed by FTase in the presence of either FPP or analogs 1-4 (Table 1) using the in vitro farnesylation assay. This lack of modification suggests the following possibilities in vivo: an enzyme other than FTase prenylates annexin; more sequence than the mimetic CaaX peptide is necessary for prenylation; and/or detection was an artifact. Two other protein substrates identified as FTase substrates in cellulo using analog $\mathbf{1}$ have CaaX sequences of CVLL ${ }^{21,22}$ and CSVL $^{21}$ and are likely modified by GGTase-I rather than FTase. In vitro these two peptides (TKCVLL and TKCSVL) showed low multiple turnover activity $\left(k_{\text {cat }} / K_{\mathrm{M}}<0.2 \mathrm{mM}^{-1} \mathrm{~s}^{-1}\right)$ catalyzed by FTase in the presence of FPP, suggesting that the comparable proteins are not readily farnesylated in cells. A similar minimal activity $\left(k_{\text {cat }} / K_{\mathrm{M}}<0.1 \mathrm{mM}^{-1} \mathrm{~s}^{-1}\right)$ was also observed with all four analogs (Table 1). Analog 1 has previously been shown to be an in vitro substrate for GGTase- $\mathrm{I}^{27}$ and this may explain the observed in cellulo activity for this and the other analogs.

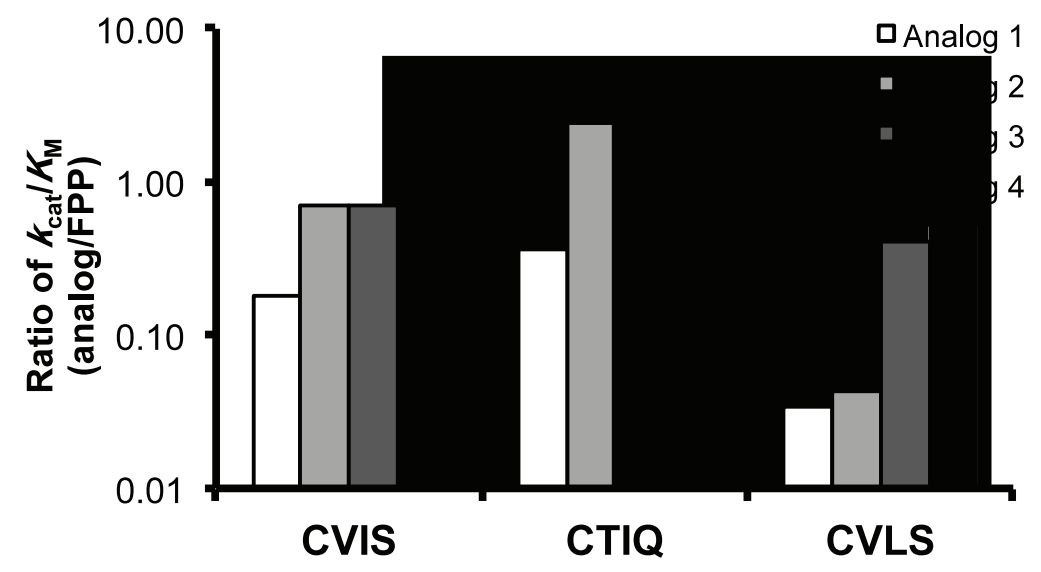

Figure 3. Relative reactivity of analogs for select dansyl-TKCxxx peptides determined as outlined in Table 1.

The results from the in vitro farnesylation assay using expected substrates (TKCCIQ and TKCVLS) ${ }^{13,21}$ demonstrated that the peptide substrate selectivity depends on the structure of both the FPP analog and the peptide. Following the same trend as the previous library, analog $\mathbf{4}$ displayed a similar reactivity pattern as FPP (Table 1). In contrast the other analogs alter substrate selectivity. For 
example, FTase did not catalyze modification of the TKCCIQ peptide with analogs $\mathbf{2}$ and $\mathbf{3}$ while TKCVLS reacted slowly with analogs 1 and $\mathbf{2}$. These results indicate that reactivity is affected by both the size of the $\mathrm{a}_{2}$ residue ( $167 \AA^{3}$ for both isoleucine and leucine) and the identity of residues at other positions. This is not unexpected as it has been previously demonstrated that the rates of farnesylation are also dependent on the identity of the amino acid in the " $\mathrm{X}$ " position. ${ }^{24}$

The last set of substrates tested were those that have been previously shown by radiolabeling ${ }^{32}$ or selective inhibition ${ }^{33}$ to be farnesylated in vivo but were not detected in large prenyl proteomic studies using analogs. Peptides representing these substrates (TKCVIS and TKCTIQ) were both modified by FPP in the in vitro assay, verifying the previous findings that they are readily farnesylated. $^{32,33}$ These substrates were also both modified by analogs $\mathbf{1}$ and $\mathbf{4}$ in the presence of FTase (Fig. 3). FTase catalyzed the reactions of both analogs $\mathbf{2}$ and $\mathbf{3}$ with TKCVIS, however only analog 2 , but not analog 3, reacted with TKCTIQ. Unexpectedly, FTase incorporated analogs 2 and $\mathbf{4}$ into TKCTIQ with a higher value of $k_{\text {cat }} / K_{\mathrm{M}}$ than FPP (Fig. 3) whereas with nearly all other peptides, FPP was the best substrate. Further studies will need to be completed to determine a mechanistic explanation for these results.

In light of the data presented here, a few trends emerge after analyzing the proteins identified when these analogs were used for in cellulo FTase substrate mapping. As previously mentioned, ${ }^{27}$ Analog 1 is also a substrate for GGTase-I consistent with the result that many of the identified proteins had CaaX sequences ending in leucine $(\mathrm{CaaL}){ }^{21,22}$ Similarly, both analogs $\mathbf{1}$ and $\mathbf{4}$ are likely substrates for RabGGTase (GGTase-II) given that several of the identified proteins ended in -xxCC or $\mathrm{xCxC}{ }^{16,21,22}$ Of those substrates likely to be FTase substrates based on identity of the terminal X residue, most contained a leucine, isoleucine, or valine residue in the $\mathrm{a}_{2}$ position. This is consistent with our in vitro data (Fig. 2) that these residues displayed the highest reactivity with the analogs. Finally, it is noted that many of FTase substrates identified from in cellulo studies are highly abundant proteins in transformed cell lines (lamin B, lamin A/C, Kras). ${ }^{16,21,22}$ Their high abundance likely off-set the decrease in reactivity observed with FTase analogs in vitro (Fig. 2 and Table 1) and thus, aided their labeling and identification.

Together, these data provide important insights relevant to the use of alkyne-modified FPP analogs to detect endogenously farnesylated proteins. To capture as many farnesylated proteins as possible, a variety of analogs should be employed in any given study to overcome analog bias. Analogs 1 and 4 displayed a roughly constant fold decrease relative to FPP (Fig. 2) suggesting they may best preserve in cellulo FTase substrate ratios. The data presented here indicate that analog $\mathbf{1}$ best mimics the peptide substrate selectivity of native FPP, whereas analog 4 is also a good mimic but only for 
substrates with $a_{2}$ residues less than $170 \AA^{3}$. Both analogs $\mathbf{2}$ and $\mathbf{3}$ are more highly variable in the ability of FTase to catalyze prenylation of a wide variety of peptide substrates. For a limited number of sequences tested, alkyne-modified analogs reacted with peptides with higher values of $k_{\text {cat }} / K_{\mathrm{M}}$ than FPP. For example, analog 3 reactivity was greater than FPP when $\mathrm{a}_{2}$ residues were very small (Gly, Ala) or very large (Phe, Trp) suggesting analog 3 may be useful in identifying farnesylation of this subset of proteins.

The present study was limited to alkyne-modified isoprenoid analogs, however the variability in reactivity and specificity seen here likely also applies to prenyl analogs modified with other functional

groups including azides ${ }^{13,17,18}$, fluorescent probes ${ }^{12}$, or biotin. ${ }^{19}$ Based on the evidence presented here, a variety of analogs is recommended for prenylomic studies, as no single analog recapitulates the specificity and reactivity of FPP. Also, alternative utilization of analogs by GGTase-I or RabGGTase should be determined and factored in when analyzing prenylomic studies. For monitoring in cellulo farnesylation of specific proteins of interest, it is recommended that multiple analogs be initially screened for labeling of that specific protein or sequence and that the highest incorporated analog be used for subsequent experiments. Alternatively, attaching the CaaX sequence or entire C-terminal domain of interest to newly developed reporter constructs allows for in cellulo tracking of prenylation with native FPP. ${ }^{34,35}$

\section{ACKNOWLEDGEMENTS}

This work was supported by NIH grants F32GM1 12317 (B.C.J.), R01GM040602 (C.A.F.), R01CA078819 (R.A.G.), R01GM084152 (M.D.D.) and NSF grant CHE-1308655 (M.D.D.). We thank Andrew Placzek for providing analogs 2 and 3. The authors publish this manuscript in memoriam to the late Richard Gibbs, who sadly passed away before its completion. 


\section{REFERENCES AND NOTES:}

1. Zverina, E. A.; Lamphear, C. L.; Wright, E. N.; Fierke, C. A. Curr. Opin. Chem. Biol. 2012, $16,544$.

2. Zhang, F. L.; Casey, P. J. Annu. Rev. Biochem. 1996, 65, 241.

3. Prior, I. A.; Lewis, P. D.; Mattos, C. Cancer Res. 2012, 72, 2457.

4. Cox, A. D.; Der, C. J. Biochim. Biophys. Acta 1997, 1333, F51.

5. Basso, A. D.; Kirschmeier, P.; Bishop, W. R. J. Lipid Res. 2006, 47, 15.

6. Whyte, D. B.; Kirschmeier, P.; Hockenberry, T. N.; Nunez-Oliva, I.; James, L.; Catino, J. J.; Bishop, W. R.; Pai, J.-K. J. Biol. Chem. 1997, 272, 14459.

7. Farrell, F. X.; Yamamoto, K.; Lapetina, E. G. Biochem. J. 1993, 289, 349.

8. Caplin, B. E.; Hettich, L. A.; Marshall, M. S. Biochim. Biophys. Acta BBA - Protein Struct. Mol. Enzymol. 1994, 1205, 39.

9. Maurer-Stroh, S.; Koranda, M.; Benetka, W.; Schneider, G.; Sirota, F. L.; Eisenhaber, F. PLoS Comput. Biol. 2007, 3.

10. London, N.; Lamphear, C. L.; Hougland, J. L.; Fierke, C. A.; Schueler-Furman, O. PLoS Comput. Biol. 2011, 7, e1002170.

11. Hicks, K. A.; Hartman, H. L.; Fierke, C. A. Biochemistry (Mosc.) 2005, 44, 15325.

12. Hannoush, R. N.; Sun, J. Nat. Chem. Biol. 2010, 6, 498.

13. Kho, Y.; Kim, S. C.; Jiang, C.; Barma, D.; Kwon, S. W.; Cheng, J.; Jaunbergs, J.; Weinbaum, C.; Tamanoi, F.; Falck, J.; Zhao, Y. Proc. Natl. Acad. Sci. U. S. A. 2004, 101, 12479.

14. Duckworth, B. P.; Zhang, Z.; Hosokawa, A.; Distefano, M. D. ChemBioChem 2007, 8, 98.

15. Wollack, J. W.; Silverman, J. M.; Petzold, C. J.; Mougous, J. D.; Distefano, M. D. ChemBioChem 2009, 10, 2934.

16. DeGraw, A. J.; Palsuledesai, C.; Ochocki, J. D.; Dozier, J. K.; Lenevich, S.; Rashidian, M.; Distefano, M. D. Chem. Biol. Drug Des. 2010, 76, 460.

17. Chan, L. N.; Hart, C.; Guo, L.; Nyberg, T.; Davies, B. S. J.; Fong, L. G.; Young, S. G.; Agnew, B. J.; Tamanoi, F. Electrophoresis 2009, 30, 3598.

18. Rose, M. w.; Rose, N. d.; Boggs, J.; Lenevich, S.; Xu, J.; Barany, G.; Distefano, M. d. J. Pept. Res. 2005, 65, 529.

19. Nguyen, U. T. T.; Guo, Z.; Delon, C.; Wu, Y.; Deraeve, C.; Fränzel, B.; Bon, R. S.; Blankenfeldt, W.; Goody, R. S.; Waldmann, H.; Wolters, D.; Alexandrov, K. Nat. Chem. Biol. 2009, 5, 227.

20. Onono, F. O.; Morgan, M. A.; Spielmann, H. P.; Andres, D. A.; Subramanian, T.; Ganser, A.; Reuter, C. W. M. Mol. Cell. Proteomics 2010, 9, 742.

21. Charron, G.; Li, M. M. H.; MacDonald, M. R.; Hang, H. C. Proc. Natl. Acad. Sci. U. S. A. 2013, 110, 11085.

22. Palsuledesai, C. C.; Ochocki, J. D.; Markowski, T. W.; Distefano, M. D. Mol. Biosyst. 2014, $10,1094$.

23. Long, S. B.; Casey, P. J.; Beese, L. S. Nature 2002, 419, 645.

24. Hougland, J. L.; Lamphear, C. L.; Scott, S. A.; Gibbs, R. A.; Fierke, C. A. Biochemistry (Mosc.) 2009, 48, 1691.

25. Reigard, S. A.; Zahn, T. J.; Haworth, K. B.; Hicks, K. A.; Fierke, C. A.; Gibbs, R. A. Biochemistry (Mosc.) 2005, 44, 11214.

26. Krzysiak, A. J.; Rawat, D. S.; Scott, S. A.; Pais, J. E.; Handley, M.; Harrison, M. L.; Fierke, C. A.; Gibbs, R. A. ACS Chem. Biol. 2007, 2, 385.

27. Hosokawa, A.; Wollack, J. W.; Zhang, Z.; Chen, L.; Barany, G.; Distefano, M. D. Int. J. Pept. Res. Ther. 2007, 13, 345.

28. Placzek, A. T.; Gibbs, R. A. Org. Lett. 2011, 13, 3576.

29. Recombinant rat FTase heterodimer was purify from E. coli as previously described. ${ }^{30}$ Dansylated peptides were purchased from Sigma and FPP was from either Sigma or Echelon Biosciences 
Incorporated. A continuous spectrofluorimetric assay was used to monitor in vitro farnesylation of peptides by detecting the change in dansyl fluorescence resulting from an increased hydrophobic environment following incorporation of farnesylated peptides into micelles. Assays were performed as previously described ${ }^{30}$ except that $0.2 \%$ detergent, $n$-octyl- $\beta$-D-glucoside (Fluka) or nonidet P-40 (Shell), was included to keep micelle hydrophobicity and concentration consistent between different analogs and FPP. Reactions were started by addition of FTase and $10 \mu \mathrm{M}$ FPP or analog (final) and the fluorescence (ex. $340 \mathrm{~nm}$ and em. $520 \mathrm{~nm}$ ) measured every $60 \mathrm{sec}$ until reactions reached completion. Following subtraction of background fluorescence, kcat/KM values were calculated as described. ${ }^{30}$

30. Hougland, J. L.; Hicks, K. A.; Hartman, H. L.; Kelly, R. A.; Watt, T. J.; Fierke, C. A. J. Mol. Biol. 2010, 395, 176.

31. Zamyatnin, A. A. Prog. Biophys. Mol. Biol. 1972, 24, 107.

32. Lai, R. K.; Perez-Sala, D.; Canada, F. J.; Rando, R. R. Proc. Natl. Acad. Sci. U. S. A. 1990, 87, 7673.

33. Vargiu, P.; Abajo, R. D.; Garcia-Ranea, J. A.; Valencia, A.; Santisteban, P.; Crespo, P.; Bernal, J. Oncogene 2004, 23, 559.

34. Flynn, S. C.; Lindgren, D. E.; Hougland, J. L. ChemBioChem 2014, 15, 2205.

35. Stein, V.; Kubala, M. H.; Steen, J.; Grimmond, S. M.; Alexandrov, K. PLoS ONE 2015, 10, e0120716. 